\title{
Dental post-operative sensitivity associated with a gallium-based restorative material
}

\author{
S. M. Dunne, ' and R. Abraham, ${ }^{2}$
}

Introduction This study forms part of a 2-year longitudinal clinical trial to compare the performance of a gallium-based restorative material (Galloy) with a high copper, mercury based (Dispersalloy) control material.

Method Following Ethical Committee approval, 25 galloy restorations and 25 Dispersalloy controls were placed in 14 adult patients, by a single operator. The cavities were of moderate size, indicating the use of amalgam as the restorative material. All restorations were polished within 1 week of placement, photographed and a silicone impression of the tooth and restoration recorded. In addition, a visual analogue scale (VAS), indicating the extent of any post-operative sensitivity, was completed by each patient for each restoration, immediately prior to polishing. A score of 0 indicated no sensitivity, while a score of 10 indicated the greatest possible sensitivity. At 6-month recall, the VAS scores, silicone impressions and photographs were repeated.

Results The mean sensitivity scores for the galloy and Dispersalloy restorations at 1 week were $5.1(+/-3.4)$ and 1.0 $(+/-1.5)$, respectively and at 6 months, $1.8(+/-3.0)$ and 0.2 $(+/-0.1)$ respectively. The differences between these means at 1 week and at 6 months were significant $(P<0.01)$.

Conclusion Galloy restorations were associated with a much greater severity of post-operative sensitivity than Dispersalloy restorations.

The use of gallium alloys as a dental restorative material was sug1 gested by Puttkamer as long ago as $1928 .{ }^{1}$ Despite this it was not until 1956 that such an alloy was produced. ${ }^{2}$ However, little interest in this alternative to silver amalgam was shown by dentists or patients.

More recently, concern about the possible toxicity of mercury containing silver amalgam has awakened interest in mercury-free alternatives. ${ }^{3,4}$

The first gallium alloy for dental purposes ${ }^{2}$ alloyed liquid gallium with powders of $\mathrm{Ta}, \mathrm{Cr}, \mathrm{Mo}, \mathrm{In}, \mathrm{Co}, \mathrm{Ni}, \mathrm{Au}$ and some $\mathrm{Cu}-\mathrm{Sn}$ alloys. The $\mathrm{Cu}-\mathrm{Sn}$ alloys produced mixes that hardened satisfactorily. ${ }^{5}$

Waterstrat made Ga-Pd-Sn alloys and found their strength and setting expansion adequate. ${ }^{6}$

In 1990, Gallium Alloy GF (Tokurike Honten, Tokyo, Japan)

${ }^{1}$ Senior Lecturer/Honorary Consultant, ${ }^{2}$ Senior Clinical Demonstrator,

Department of Conservative Dentistry, GKT Dental Institute, Denmark Hill

Campus, King's College London, Caldecot Road, London SE5 9RW

Correspondence to: Dr S. M. Dunne, Department of Conservative Dentistry, GKT

Dental Institute, Denmark Hill Campus, King's College London, Caldecot Road,

London SE5 9RW

email:s.dunne@kcl.ac.uk

REFEREED PAPER

Received 12.10.99; accepted 15.02.00

(C) British Dental Journal 2000; 188: 310-313 was introduced to the Japanese market. By alloying Ga with Sn and In, a ternary eutectic alloy was produced with a melting point as low as $10^{\circ} \mathrm{C}$. This liquid Ga alloy was mixed with spherical alloy powder consisting of Ag (50\%), Sn (25.7\%), Cu (15\%), Pd (9\%) and $\mathrm{Zn}(0.3 \%) .{ }^{7}$ However, this material showed such marked discolouration, surface roughness and marginal breakdown that a new formulation was soon introduced. The newer material possessed a lower palladium content (2\%) and was marketed as Gallium GFII. While laboratory studies suggested that the new formulation exhibited fewer defects associated with corrosion, the setting expansion was much greater than that exhibited by silver amalgam. ${ }^{7}$ In view of this, it was not surprising that a clinical trial involving Gallium GFII ${ }^{8}$ was abandoned because of the high frequency of cracking of teeth restored with the material.

In 1994, a non-palladium gallium alloy, marketed as Galloy (Southern Dental Industries, Bayswater, Australia) was introduced in Australia. Again a spherical high copper alloy powder and a Ga alloy liquid was used. The manufacturer claimed a much lower setting expansion and improved clinical performance as a result of changes in formulation and recommended procedure. Despite the manufacturer's recommendation that Galloy is indicated for use in conservative Class I and Class II posterior restorations, no restrictions on its use in moderate or larger cavities have been placed.

This most recent formulation was examined by Osborne and Summit in a clinical study and a laboratory study. ${ }^{9 a, b}$ These author's reported no major problems associated with Galloy restorations other than tarnish and surface roughness. The need to gain familiarity with the technique and the strict requirement to seal the restoration against fluid contamination, were emphasised. However, the 30 restorations placed in the clinical study were very small Class I restorations only and the author's observed that the core of knowledge for the gallium alloy restoratives was limited and that studies on Class II and extensive restorations should be conducted.

The present study attempts to enhance this core of knowledge and forms part of a 2-year longitudinal clinical trial to compare the clinical performance of Galloy with Dispersalloy (Dispersalloy, Dentsply, Milford, USA; control in the study) restorations in larger Class I and Class II cavities.

The research was supported by Southern Dental Industries (SDI) and Oral B.

\section{Method}

The authors received detailed one-to-one instruction in the use of Galloy and the delivery system by an expert nominated by SDI. The alloy and delivery system are illustrated in Figure 1. Cavities prepared in natural teeth held in a manikin were repeatedly filled using Galloy until confidence with the technique was gained.

Following Ethical Committee approval, the provision of patient information sheets and signing of patient consent forms, 25 Galloy 


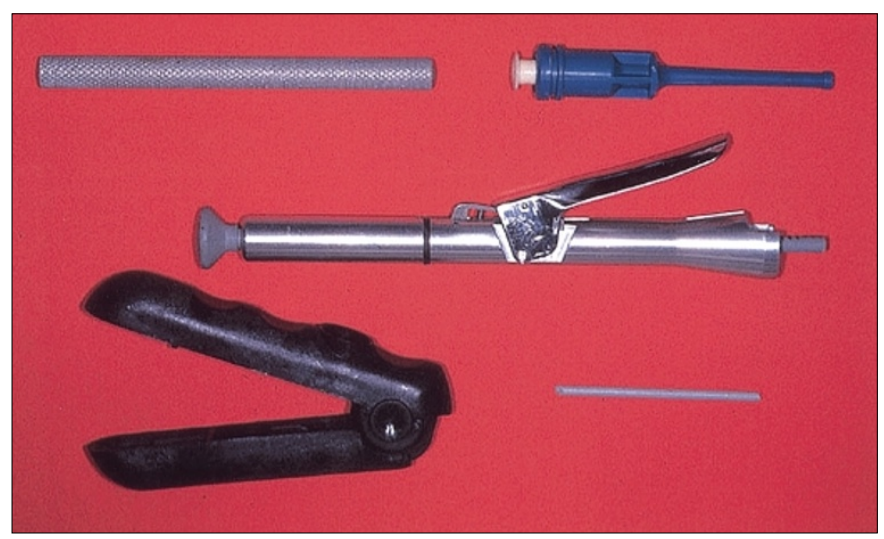

Fig. I The Galloy material and delivery system. Following trituration, the capsule is loaded into the side-action gun and the Galloy dispensed directly from the nozzle of the capsule

restorations and 25 Dispersalloy controls were placed in 14 adult patients, by a single operator (Figure 2). The patient's selected needed two or more moderate to large restorations, because of new carious lesions or failed existing restorations. The cavities were of moderate size, indicating the use of amalgam as the restorative material. The Galloy restorations consisted of 2 Class Is, 18 Class IIs (MO or DO), 2 MODs and 3 pin-retained restorations. The Dispersalloy restorations consisted of 3 Class Is, 18 Class IIs and 4 MODs. A rubber dam was used throughout. All preparations received a Vitrebond (Vitrebond, 3M, St Paul, MN, USA) resin modified glass ionomer lining. Prior to Galloy placement, all the cavities were coated with a film of low viscocity resin, supplied by the manufacturer. No resin was used in cavities to be restored with Dispersalloy. After carving, the Galloy was covered with an additional film of resin, strictly in accordance with the manufacturer's instructions. The occlusion was checked and if requiring modification, resin was reapplied to the Galloy surface. Again, no resin was applied to the surface of the Dispersalloy restorations. All restorations were polished within 1 week of placement, as recommended by Osborne and Summit. ${ }^{9 a, b}$ The restorations were then photographed and a silicone impression of the tooth and restoration recorded. In addition, the subjective severity of any post-operative discomfort was assessed for each patient, for each restoration, immediately prior to polishing. The level of discomfort was marked by the patient on a $10 \mathrm{~cm}$ visual analogue scale (VAS). ${ }^{10}$ The scale was graded 0 to 10 . A score of 0 indicated no sensitivity, while a score of 10 indicated the greatest possible sensitivity. A verbal explanation of the use of the scale preceded each assessment.

At 6-month recall, the VAS scores, silicone impressions and photographs were repeated.

Student's $t$-test was used for the statistical analysis.
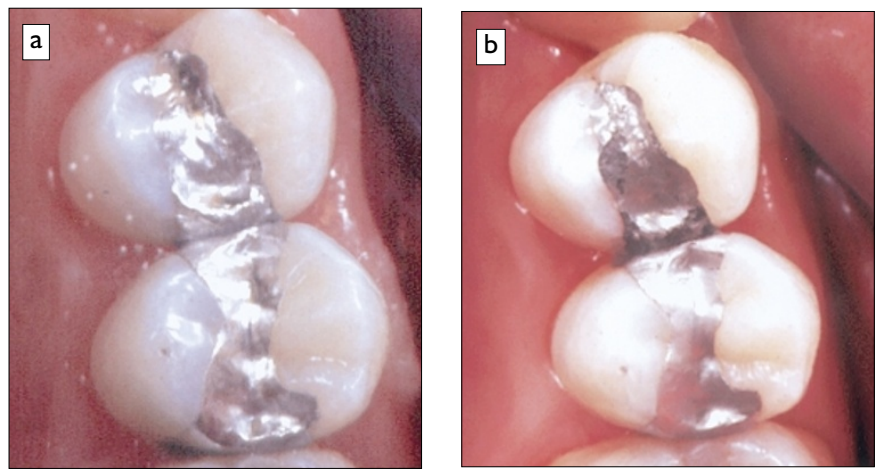

Figure 2 (a) Typical Galloy restoration UL4 (24) and Dispersalloy control UL5 (25) at I week and (b) 6 months after placement. Note the deterioration of the Galloy restoration

\section{Results}

The handling characteristics of the Galloy restorative were slightly inferior to the Dispersalloy controls. The fresh mix of Galloy was initially very plastic, allowing the alloy to stick to the condensing instrument. After initial practice, the SDI injection delivery system (see Figure 1) was easily mastered. The gallium alloy left a dark residue on latex gloves if handled.

Cavity preparations were uneventful. No pulpal exposures or pre-existing cusp fractures were noted by the operator.

The post-operative sensitivity scores for the Galloy and Dispersalloy restorations at 1 week and 6 months are included in Table 1. The mean sensitivity scores for the Galloy and Dispersalloy restorations at 1 week were $5.1(+/-3.4)$ and $1.0(+/-1.5)$, respectively and at 6 months, $1.8(+/-3.0)$ and $0.2(+/-0.1)$ respectively. The difference between these means at 1 week and at 6 months were significant $(P<0.01)$.

Two Galloy restorations were removed at the patient's request as a result of extreme and persistent sensitivity. These restorations and their Dispersalloy control were thus unavailable for VAS scoring at 6 months. A further 1 Galloy and its Dispersalloy control were lost from the study shortly after the 6-month review, at the patient's request following fracture of a tooth cusp associated with the Galloy restoration.

By the 6-month recall, four Galloy restorations were associated with a tooth cusp fracture (Figure 3 ). All of the restorations associated with a cusp fracture remained in situ at this time and the damaged tooth structure was repaired using glass ionomer cement. Thus, they were not lost from the study at 6 months.

\section{Discussion}

Gallium is a metal which belongs to the group IIIa elements of the periodic table and occurs in small amounts in a variety of minerals. It is present in all aluminium ores and accounts for some $15 \mathrm{~g}$ per ton of the earth's crust. It is a soft silvery metal with a brilliant lustre which tarnishes rapidly with the formation of a thin oxide film.

Table I. VAS scores for post-operative sensitivity, I week and 6 months after placement of Galloy and Dispersalloy restorations (dis $=$ discontinued). The differences between the means at I week and at 6 months were significant $(P<0.0 I)$

\begin{tabular}{ccc}
\hline I wk & Galloy scores (0-10) & Dispersalloy \\
$6 \mathrm{mth}$ & $\mathrm{I}$ wk $6 \mathrm{mth}$ \\
\hline
\end{tabular}

\begin{tabular}{|c|c|c|c|c|}
\hline & $\begin{array}{l}7 \\
8 \\
6 \\
9 \\
9 \\
3.5 \\
7.5 \\
9 \\
9 \\
2 \\
3 \\
3 \\
3 \\
3 \\
8.5 \\
8.5 \\
7 \\
0 \\
0 \\
8.5 \\
4 \\
8 \\
8 \\
8 \\
0\end{array}$ & $\begin{array}{c}0 \\
0 \\
0 \\
0 \\
7.5 \\
9 \\
\text { dis } \\
1.5 \\
7.5 \\
5 \\
5 \\
5 \\
\text { dis } \\
0 \\
2 \\
0 \\
0 \\
0 \\
0 \\
0 \\
0 \\
0 \\
0 \\
0 \\
0\end{array}$ & $\begin{array}{l}0 \\
4 \\
2 \\
3 \\
0 \\
2 \\
2 \\
6 \\
0 \\
0 \\
0 \\
0 \\
0 \\
0 \\
1 \\
0 \\
1.5 \\
0 \\
0 \\
0 \\
2 \\
0 \\
0 \\
0 \\
2.5 \\
1 \\
1 \\
0 \\
0\end{array}$ & $\begin{array}{r}0 \\
0 \\
0 \\
0 \\
0 \\
1 \\
0 \\
0 \\
0 \\
0 \\
0 \\
3 \\
\text { dis } \\
0 \\
0 \\
0 \\
0 \\
0 \\
0 \\
0 \\
0 \\
0 \\
0 \\
0 \\
0\end{array}$ \\
\hline $\begin{array}{l}\text { Mean } \\
\text { SD }\end{array}$ & $\begin{array}{l}5.1 \\
3.4\end{array}$ & $\begin{array}{l}1.8 \\
3.0\end{array}$ & $\begin{array}{l}1.0 \\
1.5\end{array}$ & $\begin{array}{l}0.2 \\
0.1\end{array}$ \\
\hline
\end{tabular}



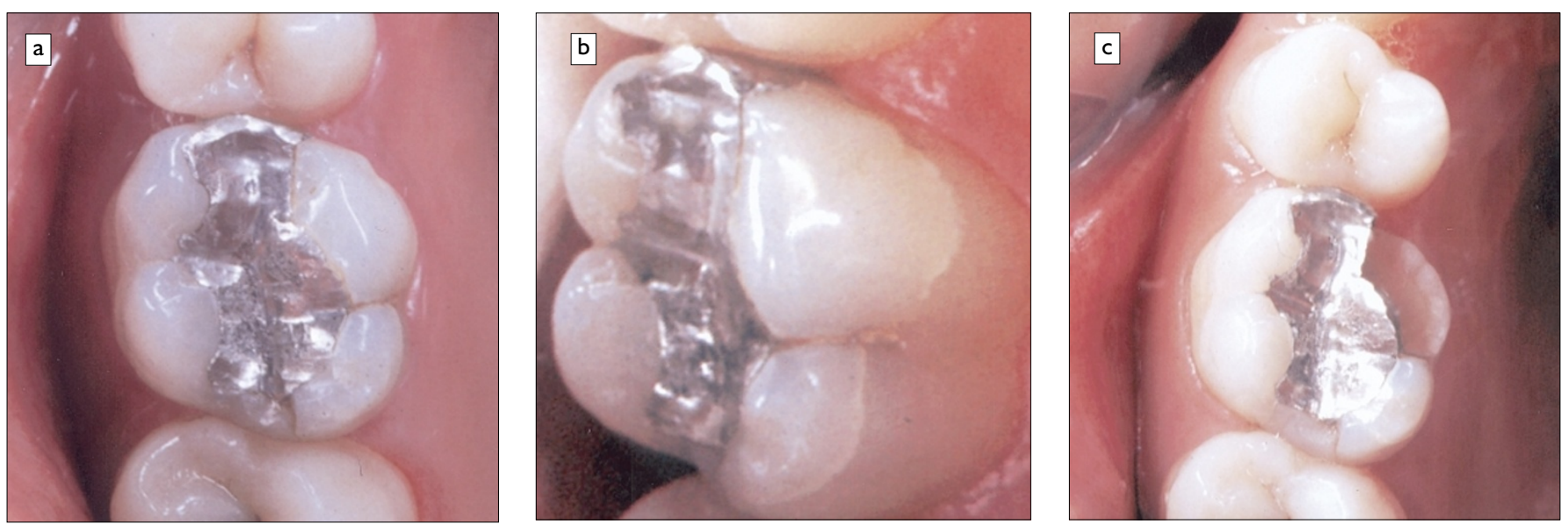

Fig. 3 a) An extensive MO Galloy restoration placed in tooth UR6(16). b) Tooth cusp fracture associated with the Galloy restoration in tooth UR6. c) Subsequent loss of the mesio-palatal cusp UR6. The defect was repaired with a glass ionomer cement and the restoration was not lost from the trial

Gallium can be made to adhere to glass to form a white mirror, and it was this 'wetting' property that was responsible for the suggestion that it might be alloyed with powdered metals to form a substitute for mercury-containing dental amalgam. ${ }^{2}$

A characteristic of gallium is that it has a low melting point $\left(29.8^{\circ} \mathrm{C}\right)$. The boiling point is high $\left(\right.$ over $\left.1,700^{\circ} \mathrm{C}\right)$ and the vapour pressure low $\left(10 \mathrm{~Pa}\right.$ at $\left.1,178^{\circ} \mathrm{C}\right)$. The density of liquid gallium is $6.09 \mathrm{~g} / \mathrm{cm}^{3}$ compared with $13.55 \mathrm{~g} / \mathrm{cm}^{3}$ for mercury. ${ }^{7}$

Gallium has been used for many years in the electronics industry as a compound semiconductor and also in medicine as a chemotherapeutic agent in the treatment of cancer. ${ }^{11}$

In the manner of the mercury toxicity debate ${ }^{3,4}$ the biocompatability of gallium alloys has been a matter of concern for more than 30 years. Early studies reported severe foreign body reactions to subcutaneous implants of gallium alloys. ${ }^{12}$ However, these alloys contained metals likely to maximise the tissue response. More recent studies have shown tissue responses less severe than with mercury-containing silver amalgam. ${ }^{13}$ However, gallium ions severely disrupt cellular metabolism ${ }^{14}$ and this effect has been used in anti-cancer drugs, as mentioned above. In a review of gallium alloys as restorative materials, Hero and Okabe ${ }^{7}$ considered the biocompatibility of gallium alloys to be still controversial and that further studies were needed.

Clinical reports on the use of gallium-based dental alloys are few. Early materials showed excessive setting expansion, tarnish and corrosion. ${ }^{7}$ However, apart from surface tarnish and roughness, no major problems associated with Galloy were reported by Osborne and Summitt. ${ }^{9 a, b}$

The composition of the Galloy powder is $\mathrm{Ag}(60.1 \%)$, $\mathrm{Sn}(28.05 \%), \mathrm{Cu}(11.8 \%)$ and $\mathrm{Pt}(0.05 \%)$. The liquid contains $\mathrm{Ga}(62 \%)$, In (25\%), Sn (13\%) and $\mathrm{Bi}(0.05 \%) .{ }^{7}$ The alloying of $\mathrm{Ga}$ with In and $\mathrm{Sn}$ brings the melting point down from $29.8^{\circ} \mathrm{C}$ for pure $\mathrm{Ga}$ to about $10^{\circ} \mathrm{C}$, rendering it suitable for dental purposes. The setting expansion is documented as well within ISO limits and creep resistance and $1 \mathrm{hr}$ compressive strength have been found to be well above minimum requirements. ${ }^{7}$

The manufacturer states that free $\mathrm{Ga}$ is present in the restoration for up to $18 \mathrm{hrs}$ after placement and recommends application of a water-insoluble cavity liner to prevent corrosion reactions (and consequently excessive expansion) with the free Ga. For the same reason, an additional layer of resin is to be applied to the surface of the finished restoration. This requirement for resin protection for each gallium restoration was strictly observed in the present study.

The delivery system (see Figure 1) and handling of the Galloy differed from that of a conventional amalgam but was easily mas- tered. This observation is in accordance with that of Osborne and Summit. ${ }^{9 a, b}$ The Galloy adherent to the condensing instrument during the early stages of condensation was easily removed. The use of the sealant under and over the Galloy restorations did not add greatly to the time devoted to the procedure. Indeed, the present authors' commonly employ bonding resins when placing amalgam restorations in their routine clinical practice.

The VAS scale was selected for the subjective assessment of post-operative sensitivity. One of the author's was experienced in the use of such scales ${ }^{15}$ and their use has been recommended in the assessment of dental pain. ${ }^{16}$ The VAS offers a continuum between extremes of pain and has the capacity to indicate a small change in severity. ${ }^{17}$ Moreover, a very high correlation between successive measurements of pain severity has been reported with the VAS. ${ }^{18}$

Despite the great care taken in the placement of all restorations and, in particular, the avoidance of moisture contamination of the Galloy restorations, the incidence and severity of post-operative sensitivity were much higher in the Galloy restorations in comparison to the Dispersalloy controls. Indeed, two Galloy restorations were removed at the patient's request as a result of extreme and persistent sensitivity and were unavailable for VAS scoring at 6 months. The sensitivity associated with the Galloy restorations improved in the majority of cases by the 6-month recall. However, sensitivity increased in six cases. This is in marked contrast to the findings of Osborne and Summit ${ }^{9 a}, \mathrm{~b}$ where, although it was not specifically investigated, no post-operative sensitivity was reported. The present authors suggest that these markedly different results are explained by the differences in cavity size and types in the two studies. The very small volume of restorative material and the integrity of the surrounding tooth structure in the Osborne and Summit study ${ }^{9 a, b}$ contrasted with the larger restorations and thus weaker tooth-structure in the present study. The lack of control restorations in the former study also complicates interpretation of the results.

By the 6-month recall, four (17\%) Galloy restorations were associated with a related tooth cusp fracture (see Figure 3). In contrast, no Dispersalloy restorations were associated with a similar fracture. Again, this result is not in accordance with those of Osborne and Summit. ${ }^{9 a, b}$

The fresh mix of Galloy was initially very plastic. Thus, no excessive forces were required to be generated during packing. It is, therefore, unlikely that the observation of subsequent enamel cracks could be blamed on packing technique. The present authors' attribute both the marked post-operative sensitivity and the high 
incidence of tooth cusp fracture associated with Galloy restorations to excessive expansion of the gallium alloy. This is in accordance with the research of Blair et al. ${ }^{19}$ where the expansion of gallium alloys was found to exceed the ISO standard for amalgam. The latter authors concluded that there was a potential for splitting teeth in a clinical situation. Despite the extensive measures taken to prevent moisture contamination, in the oral environment, post-operative expansion of the gallium-based alloy, in larger Class I and Class II cavities, remains unacceptably high.

The laboratory-based data published by the manufacturer does not appear to be a good predictor of clinical performance in moderate and large restorations. However, the manufacturer's advertising literature states that Galloy is indicated for conservative Class I and II posterior restorations.

It is possible that the application of resin under and over the Galloy restoration did not form a complete barrier to moisture contamination of the setting (and free-gallium rich) restoration. Indeed, it is unlikely that the proximal surface of Class II restorations could ever be completely covered with resin where an adjacent tooth is present. This is in contrast to the study of Osborne and Summitt ${ }^{9 a, b}$ where the small Class I restorations could be sealed thoroughly, on all surfaces.

The gallium alloy left a dark residue on latex gloves if handled. It is possible that some patients might be concerned by the appearance of dirty gloves after their treatment.

\section{Conclusions}

Galloy restorations were associated with a much greater incidence and severity of post-operative sensitivity than Dispersalloy control restorations. In addition, the high incidence of tooth fracture associated with larger Class I and Class II Galloy restorations gives cause for concern. Even at this early phase of the study, the authors cannot recommend the use of Galloy in moderate to large Class I and Class II cavities. The longitudinal study continues and further results, including longer term data on surface and marginal degradation will be forthcoming. Investigations of the nature of the problems associated with Galloy and methods of limiting such problems are underway.
1 Puttkammer A. Mercury-free amalgam? Zahnaerztl Rundsch 1928; 35 : 1450-1454.

2 Smith D L, Caul H J. Alloys of gallium with powdered metals as possible replacements for dental amalgam. J Am Dent Assoc 1956; 53: 315-324.

3 Dunne S M, Wilson N, Gainsford I D. Current material and techniques for direct placement restorations in posterior teeth. Part 1: Silver amalgam. Int Dent J 1997; 47:123-136.

4 Wilson N, Dunne S M, Gainsford I D. Current material and techniques for direct placement restorations in posterior teeth. Part 2: Composites. Int Dent J 1997; 47: 185-193.

5 Smith D L, Caul H J, Sweeney W T. Some physical properties of galliumcopper-tin alloys. J Am Dent Assoc 1956; 53: 677-685.

6 Waterstrat R M. Evaluation of gallium-palladium-tin alloy for restorative dentistry. I Am Dent Assoc 1969; 78: 536-541.

7 Hero H, Okabe T. Gallium alloys as dental restorative materials: A research review. Cells and Materials 1994; 4: 409-418.

8 Navarro M, Franco E, Bastos P, Carvalho R, Teixera L. Clinical evaluation of gallium alloy. J Dent Res 1993; 72: 219.

9a Osborne J W, Summitt J B. Mechanical properties and clinical performance of a gallium restorative material. Oper Dent 1995; 20: 241-245.

9 b Osborne J W, Summitt J B. 2-year clinical evaluation of a gallium restorative alloy. Am J Dent 1996; 9: 191-194.

10 Huskisson E C. Visual analogue scales. In: R Melzak (Ed) Pain measurements and assessment. New York: Raven Press, 1983, pp33-37.

11 Hall T J, Chambers T J. Gallium inhibits bone resorption by a direct effect on osteoclasts. Bone Miner 1990; 8: 211-216.

12 Waterstrat R M, Longton R W. Gallium-palladium alloys as dental filling materials. Pub Health Rep 1964; 79: 638-643.

13 Kaga M, Ohkawa S, Hanawa T, Oguchi H. Comparative evaluation of biocompatibility of gallium alloy and amalgams in rats subcutaneous tissue. J Jap Soc Dent Mat 1992; 11: 740-745.

14 Aoki Y, Lipsky M M, Fowler B A. Alteration in protein synthesis in primary cultures of rat kidney proximal tubule epithelial cells by exposure to gallium, indium and arsenite. Toxicol Appl Pharmacol 1990; 106: 462-468.

15 Dunne S M, Hannington-Kiff J G. The use of topical guanethidine in the treatment of dentine hypersensitivity: A controlled study. Pain 1993; 54: 165-168.

16 Clark G E, Troullos E S. Designing hypersensitivity clinical studies. In: F A Curro (ed) Tooth hypersensitivity. Dent Clin N Am, Philadelphia: Saunders, 1990,pp ix.

17 Deschamps M, Band P R, Goldman A J. Assessment of adult cancer pain: shortcomings of current methods. Pain 1988; 32: 133-139.

18 Scott J, Huskisson E C. Accuracy of subjective measurements made with or without previous scores; an important source of error in serial measurements of subjective states. Ann Rheum Dis 1979; 38: 558-559.

19 Blair F M, Whitworth J M, McCabe J F. The physical properties of a gallium alloy restorative material. Dent Mater 1995; 11: 277-280. 